\title{
Existence and Continuous Dependence for Fractional Partial Hyperbolic Differential Equations
}

\author{
Qixiang Dong, ${ }^{1}$ Guangxian $\mathrm{Wu}^{2}$ and Lanping $\mathrm{Zhu}^{1}$ \\ ${ }^{1}$ School of Mathematical Sciences, Yangzhou University, Yangzhou 225002, China \\ ${ }^{2}$ Department of Mathematics, Jiaozuo Teachers College, Jiaozuo 454001, China \\ Correspondence should be addressed to Qixiang Dong; qxdongyz@outlook.com
}

Received 20 June 2015; Accepted 29 November 2015

Academic Editor: Hugo Leiva

Copyright (c) 2015 Qixiang Dong et al. This is an open access article distributed under the Creative Commons Attribution License, which permits unrestricted use, distribution, and reproduction in any medium, provided the original work is properly cited.

\begin{abstract}
This paper is concerned with a class of fractional hyperbolic partial differential equations with the Caputo derivative. Existence and continuous dependence results of solutions are obtained under the hypothesis of the Lipschitz condition without any restriction on the Lipschitz constant. Examples are discussed to illustrate the results.
\end{abstract}

\section{Introduction}

This paper is mainly concerned with the fractional order hyperbolic functional differential equation

$$
\begin{aligned}
& \left({ }^{c} D_{0}^{r} u\right)(x, y)=f\left(x, y, u_{(x, y)}\right), \\
& (x, y) \in J:=[0, a] \times[0, b], \\
& u(x, y)=\phi(x, y), \\
& (x, y) \in \widetilde{J}:=[-\alpha, a] \times[-\beta, b] \backslash(0, a] \times(0, b], \\
& u(x, 0)=\varphi(x), \quad x \in[0, a], \\
& u(0, y)=\psi(y), \quad y \in[0, b],
\end{aligned}
$$

where $\alpha, \beta, a, b>0,{ }^{c} D_{0}^{r}$ is the standard Caputo fractional derivative of order $r=\left(r_{1}, r_{2}\right) \in(0,1] \times(0,1], f: J \times$ $C \rightarrow \mathbb{R}^{n}$ is a given function, $\phi: \widetilde{J} \rightarrow \mathbb{R}^{n}$ is a given continuous function, $\varphi:[0, a] \rightarrow \mathbb{R}^{n}, \psi:[0, b] \rightarrow \mathbb{R}^{n}$ are given absolutely continuous functions with $\varphi(x)=\phi(x, 0)$, $\psi(y)=\phi(0, y)$ for each $x \in[0, a]$ and $y \in[0, b]$, and $C:=$ $C\left([-\alpha, 0] \times[-\beta, 0] ; \mathbb{R}^{n}\right)$ is the space of continuous functions on $[-\alpha, 0] \times[-\beta, 0]$.
Fractional differential equations have been widely and efficiently used to describe many phenomena arising in engineering, physics, economy, and science ([1-3]). For example, it was showed by Hilfer that time fractional derivatives are equivalent to infinitesimal generators of generalised time fractional evolution which arise in the transition from microscopic to macroscopic time scales; see, for example, $[4,5]$. More applications of fractional derivatives can be found in $[6,7]$. For this reason, the theory of differential equations of fractional order has recently received a lot of attention and now constitutes a significant branch of nonlinear analysis. Numerous research papers and monographs have appeared which are devoted to fractional differential equations. See [6, 8-12], for example, and references therein. A special issue on recent advances in fractional differential equations was introduced in [13].

In $[1,14]$, the authors studied a class of fractional hyperbolic functional differential equations. The existence results were obtained under the Lipschitz conditions, with some additional restrictions on the Lipschitz constants. In this paper, we continue to study fractional hyperbolic functional differential equation with finite delay (1)-(3). We give the existence of solutions under the assumption of Lipschitz condition without any restriction on the Lipschitz constant. As a corollary, existence for corresponding boundary value problem is obtained. Continuous dependence of solutions on 
the initial data is also studied. Some examples are presented to illustrate our results.

\section{Preliminaries}

In this section we collect some definitions and results needed in our further investigations.

Definition 1 (see $[3,6])$. Let $\alpha>0$ be a fixed number. The Riemann-Liouville fractional integral of order $\alpha>0$ of the function $h:[a, b] \rightarrow \mathbb{R}$ is defined by

$$
I_{a}^{\alpha} h(t)=\frac{1}{\Gamma(\alpha)} \int_{a}^{t}(t-s)^{\alpha-1} h(s) d s, \quad t \in[a, b]
$$

provided that the right side is pointwise defined, where $\Gamma(\cdot)$ denotes the gamma function; that is, $\Gamma(z)=\int_{0}^{\infty} e^{-t} t^{z-1} d t$.

Definition 2 (see $[3,6])$. Let $\alpha>0$ be fixed and $n=[\alpha]+1$. The Caputo fractional derivative of order $\alpha$ of $h:(a, b] \rightarrow \mathbb{R}$ at the point $t$ is defined by

$$
{ }^{c} D_{a}^{\alpha} h(t)=\frac{1}{\Gamma(n-\alpha)} \int_{a}^{t}(t-s)^{n-\alpha-1} h_{(n)}(s) d s,
$$

$$
t \in[a, b]
$$

provided that the right side is pointwise defined, where $[\alpha]$ denotes the integer part of the real number $\alpha$.

If $0<\alpha<1$, then

$$
{ }^{c} D_{a}^{\alpha} h(t)=\frac{1}{\Gamma(1-\alpha)} \int_{a}^{t}(t-s)^{\alpha-1} h^{\prime}(s) d s .
$$

We list some basic properties of fractional integral and derivative without proof. For more details, we refer the readers to $[3,6]$.

Lemma 3 (see [3]). (1) For $\alpha>0$ and $h_{1}, h_{2} \in L^{1}[a, b]$, the identity

$$
I_{a}^{\alpha}\left(c_{1} h_{1}(t)+c_{2} h_{2}(t)\right)=c_{1} I_{a}^{\alpha} h_{1}(t)+c_{2} I_{a}^{\alpha} h_{2}(t)
$$

holds for $t \in[a, b]$ and some constants $c_{1}, c_{2} \in \mathbb{R}$. If additionally $h_{1}, h_{2} \in A C^{n}[a, b]$, then

$$
{ }^{c} D_{a}^{\alpha}\left(c_{1} h_{1}(t)+c_{2} h_{2}(t)\right)=c_{1}^{c} D_{a}^{\alpha} h_{1}(t)+c_{2}^{c} D_{a}^{\alpha} h_{2}(t)
$$

for $t \in[a, b]$.

(2) If $\alpha, \beta>0$ and $h \in L^{1}[a, b]$, then

$$
I_{a}^{\alpha} I_{a}^{\beta} h(t)=I_{a}^{\alpha+\beta} h(t)
$$

holds almost everywhere for $t \in[a, b]$. If additionally $h$ is continuous on $[a, b]$, then the identity holds everywhere for $t \in[a, b]$.

(3) If $\alpha>0$ and $h \in C[a, b]$, then

$$
{ }^{c} D_{a}^{\alpha} I_{a}^{\alpha} h=h .
$$

(4) If $\alpha>0$ and $h \in C^{n}[a, b]$, then

$$
I_{a}^{\alpha c} D_{a}^{\alpha} h(t)=h(t)-\sum_{k=0}^{n-1} \frac{h^{(k)}(a)}{k !}(t-a)^{k}
$$

for $t \in[a, b]$, where $n=[\alpha]+1$ and $C^{n}[a, b]$ denotes the space of the function $f:[a, b] \rightarrow \mathbb{R}$ such that $f^{(n-1)}$ is absolutely continuous on $[a, b]$.

Definition 4 (see $[1,14])$. Let $\alpha \in(0, \infty)$ and $u \in L^{1}\left(J ; \mathbb{R}^{n}\right)$. The partial Riemann-Liouville integral of order $\alpha$ of $u(x, y)$ with respect to $x$ is defined by the expression

$$
I_{0, x}^{\alpha} u(x, y)=\frac{1}{\Gamma(\alpha)} \int_{0}^{x}(x-s)^{\alpha-1} u(s, y) d s,
$$

$$
(x, y) \in J \text {. }
$$

Analogously, we define the integral

$$
I_{0, y}^{\alpha} u(x, y)=\frac{1}{\Gamma(\alpha)} \int_{0}^{y}(y-s)^{\alpha-1} u(x, s) d s,
$$

$$
(x, y) \in J \text {. }
$$

Definition 5 (see $[1,14])$. Let $r=\left(r_{1}, r_{2}\right) \in(0, \infty) \times(0, \infty)$ and $u \in L^{1}\left(J ; \mathbb{R}^{n}\right)$. The left-side mixed Riemann-Liouville integral of order $r$ of $u$ is defined by

$$
\begin{aligned}
& \left(I_{0}^{r} u\right)(x, y)=\frac{1}{\Gamma\left(r_{1}\right) \Gamma\left(r_{2}\right)} \\
& \quad \cdot \int_{0}^{x} \int_{0}^{y}(x-s)^{r_{1}-1}(y-t)^{r_{2}-1} u(s, t) d t d s .
\end{aligned}
$$

In particular,

$$
\begin{aligned}
& \left(I_{0}^{0} u\right)(x, y)=u(x, y), \\
& \left(I_{0}^{\sigma} u\right)(x, y)=\int_{0}^{x} \int_{0}^{y} u(s, t) d t d s,
\end{aligned}
$$

where $\sigma=(1,1)$.

By $1-r$ we mean $\left(1-r_{1}, 1-r_{2}\right) \in[0,1) \times[0,1)$. Denote by $D_{x y}^{2}:=\partial^{2} / \partial x \partial y$ the mixed second-order partial derivative.

Definition 6 (see $[1,14])$. Let $r \in(0,1] \times(0,1]$ and $u \in$ $L^{1}\left(J ; \mathbb{R}^{n}\right)$. The Caputo mixed fractional order derivative of order $r$ of $u$ is defined by the expression $\left({ }^{c} D_{0}^{r} u\right)(x, y)=$ $\left(I_{0}^{1-r} D_{x y}^{2} u\right)(x, y)$; that is,

$$
\begin{aligned}
& \left({ }^{c} D_{0}^{r} u\right)(x, y)=\frac{1}{\Gamma\left(1-r_{1}\right) \Gamma\left(1-r_{2}\right)} \\
& \cdot \int_{0}^{x} \int_{0}^{y}(x-s)^{-r_{1}}(y-t)^{-r_{2}} \frac{\partial^{2}}{\partial s \partial t} u(s, t) d t d s .
\end{aligned}
$$

The case $\sigma=(1,1)$ is included and we have

$$
\left({ }^{c} D_{0}^{\sigma} u\right)(x, y)=\left(D_{x y}^{2} u\right)(x, y)=\frac{\partial^{2}}{\partial x \partial y} u(x, y) \text {. }
$$


In the sequel we need the following lemma to transform partial fractional differential equations into integral equations. We denote by $A C\left(J ; \mathbb{R}^{n}\right)$ the space of absolutely continuous functions defined on $J$.

Lemma 7 (see $[1,14])$. A function $u \in A C\left(J ; \mathbb{R}^{n}\right)$ such that its mixed derivative $D_{x y}^{2} u$ exists and is integrable on $J$ is a solution of problem

$$
\begin{aligned}
\left({ }^{c} D_{0}^{\sigma} u\right)(x, y) & =f(x, y), \quad(x, y) \in J, \\
u(x, 0) & =\varphi(x), \quad x \in[0, a], \\
u(0, y) & =\psi(y), \quad y \in[0, b], \\
\varphi(0) & =\psi(0),
\end{aligned}
$$

if and only if $u(x, y)$ satisfies

$$
u(x, y)=\mu(x, y)+\left(I_{0}^{r} f\right)(x, y), \quad(x, y) \in J,
$$

where

$$
\mu(x, y)=\varphi(x)+\psi(y)-\varphi(0) .
$$

We also need the following generalization of Gronwall's inequality for two independent variables and singular kernel.

Lemma $8($ see $[1,15])$. Let $v: J \rightarrow[0,+\infty)$ be a real function and $w(\cdot, \cdot)$ a nonnegative, locally integrable function on $\mathrm{J}$. If there are constants $c>0$ and $0<r_{1}, r_{2}<1$ such that

$$
v(x, y) \leq w(x, y)+c \int_{0}^{x} \int_{0}^{y} \frac{v(s, t)}{(x-s)^{r_{1}}(y-t)^{r_{2}}} d t d s,
$$

then there exists a constant $\delta=\delta\left(r_{1}, r_{2}\right)$ such that

$$
\begin{aligned}
v(x, y) \leq & w(x, y) \\
& +c \delta \int_{0}^{x} \int_{0}^{y} \frac{w(s, t)}{(x-s)^{r_{1}}(y-t)^{r_{2}}} d t d s .
\end{aligned}
$$

\section{Main Results}

We begin with the definition of solutions to problem (1)-(3).
Definition 9. A function $u \in C_{(a, b)}:=C\left([-\alpha, a] \times[-\beta, b] ; \mathbb{R}^{n}\right)$ is said to be a solution to (1)-(3), if its mixed derivative $D_{x y}^{2}$ exists and is integrable, (2), (3) are satisfied, and

$$
\begin{aligned}
& u(x, y)=\mu(x, y)+\frac{1}{\Gamma\left(r_{1}\right) \Gamma\left(r_{2}\right)} \\
& \cdot \int_{0}^{x} \int_{0}^{y}(x-s)^{r_{1}-1}(y-t)^{r_{2}-1} f\left(s, t, u_{(s, t)}\right) d t d s
\end{aligned}
$$

for $(x, y) \in J$, where $\mu$ is the function given by (20).

We first give an existence and uniqueness result based on the Banach contraction principle.

Theorem 10. Assume that $f: J \times C \rightarrow \mathbb{R}^{n}$ is continuous and is Lipschitz continuous with respect to the last variable; that is, there is $k>0$ such that

$$
\|f(x, y, u)-f(x, y, v)\| \leq k\|u-v\|_{C}
$$

for any $u, v \in C$ and $(x, y) \in J$. Then there exists a unique solution to (1)-(3) on $[-\alpha, a] \times[-\beta, b]$.

Proof. Let $N=\left[\left(k a^{r_{1}} b^{r_{2}} / \Gamma\left(r_{1}\right) \Gamma\left(r_{2}\right)\right)^{1 / r_{1}}\right]+1$ and $h_{i}=i a / N$, where $[\alpha]$ denote the integer part of a real number $\alpha$. Then $0=h_{0}<h_{1}<h_{2}<\cdots<h_{N}=a$, and

$$
\frac{k\left(h_{i+1}-h_{i}\right)^{r_{1}} b^{r_{2}}}{\Gamma\left(r_{1}+1\right) \Gamma\left(r_{2}+1\right)}<1
$$

for $i=1,2, \ldots, N$.

We first focus on $\left[0, h_{1}\right] \times[0, b]$. Let us define a subset $C_{h_{1}} \subset C\left(\left[-\alpha, h_{1}\right] \times[-\beta, b] ; \mathbb{R}^{n}\right)$ which contains those functions $u$ that $u(x, y)=\phi(x, y)$ for $(x, y) \in \widetilde{J}$ and the restriction of $u$ on $\left[0, h_{1}\right] \times[0, b]$ is continuous. It is easily seen that $C_{h_{1}}$ is closed in $C\left(\left[-\alpha, h_{1}\right] \times[-\beta, b] ; \mathbb{R}^{n}\right)$, and $C_{h_{1}}$ can also be considered as a closed subset in $C\left(\left[0, h_{1}\right] \times[0, b] ; \mathbb{R}^{n}\right)$. We define an operator $N: C_{h_{1}} \rightarrow C_{h_{1}}$ by

$$
(N u)(x, y)= \begin{cases}\phi(x, y), & (x, y) \in \widetilde{J}, \\ \mu(x, y)+\frac{1}{\Gamma\left(r_{1}\right) \Gamma\left(r_{2}\right)} \int_{0}^{x} \int_{0}^{y} f\left(s, t, u_{(s, t)}\right)(x-s)^{r_{1}-1}(y-t)^{r_{2}-1} d t d s, & (x, y) \in\left[0, h_{1}\right] \times[0, b] .\end{cases}
$$

One can easily verify that $N$ is well-defined and maps $C_{h_{1}}$ into itself. Further, for any $u^{\prime}, u^{\prime \prime} \in C_{h_{1}}$ and every $(x, y) \in\left[0, h_{1}\right] \times$ $[0, b]$, we have

$$
\begin{aligned}
& \left\|\left(N u^{\prime}\right)(x, y)-\left(N u^{\prime \prime}\right)(x, y)\right\| \leq \frac{1}{\Gamma\left(r_{1}\right) \Gamma\left(r_{2}\right)} \\
& \cdot \int_{0}^{x} \int_{0}^{y}\left\|f\left(s, t, u_{(s, t)}^{\prime}\right)-f\left(s, t, u_{(s, t)}^{\prime \prime}\right)\right\|(x-s)^{r_{1}-1}
\end{aligned}
$$

$$
\begin{aligned}
& \cdot(y-t)^{r_{2}-1} d t d s \leq \frac{k}{\Gamma\left(r_{1}\right) \Gamma\left(r_{2}\right)} \\
& \cdot \int_{0}^{x} \int_{0}^{y}\left\|u_{(s, t)}^{\prime}-u_{(s, t)}^{\prime \prime}\right\|_{C}(x-s)^{r_{1}-1} \\
& \cdot(y-t)^{r_{2}-1} d t d s .
\end{aligned}
$$


Noting that $u^{\prime}(x, y)=u^{\prime \prime}(x, y)$ for $x \in[-\alpha, 0]$ and $y \in$ $[-\beta, 0]$, we have

$$
\begin{aligned}
& \left\|u_{(s, t)}^{\prime}-u_{(s, t)}^{\prime \prime}\right\|_{C}=\sup _{(x, y) \in[-\alpha, 0] \times[-\beta, 0]} \| u^{\prime}(s+x, t+y) \\
& -u^{\prime \prime}(s+x, t+y)\left\|\leq \sup _{(x, y) \in\left[0, h_{1}\right] \times[0, b]}\right\| u^{\prime}(x, y) \\
& -u^{\prime \prime}(x, y)\|\leq\| u^{\prime}-u^{\prime \prime} \|_{C_{h_{1}}} .
\end{aligned}
$$

It follows that

$$
\begin{aligned}
& \left\|\left(N u^{\prime}\right)(x, y)-\left(N u^{\prime \prime}\right)(x, y)\right\| \\
& \leq \frac{k}{\Gamma\left(r_{1}\right) \Gamma\left(r_{2}\right)} \int_{0}^{x} \int_{0}^{y}(x-s)^{r_{1}-1}(y-t)^{r_{2}-1} d t d s \\
& \quad \cdot\left\|u^{\prime}-u^{\prime \prime}\right\|_{C_{h_{1}}} \\
& \leq \frac{k h_{1}^{r_{1}} b^{r_{2}}}{\Gamma\left(r_{1}+1\right) \Gamma\left(r_{2}+1\right)}\left\|u^{\prime}-u^{\prime \prime}\right\|_{C_{h_{1}}} .
\end{aligned}
$$

Take the supremum for $(x, y) \in\left[0, h_{1}\right] \times[0, b]$; we get that

$$
\begin{aligned}
& \left\|N u^{\prime}-N u^{\prime \prime}\right\|_{C_{h_{1}}} \\
& \leq \frac{k h_{1}^{r_{1}} b^{r_{2}}}{\Gamma\left(r_{1}+1\right) \Gamma\left(r_{2}+1\right)}\left\|u^{\prime}-u^{\prime \prime}\right\|_{C_{h_{1}}} .
\end{aligned}
$$

From (25) we know that $N$ is a contraction on $C_{h_{1}}$. An application of Banach contraction principle yields a unique fixed point of $N$ in $C_{h_{1}}$, which is the unique solution of (1)(3) on $\left[-\alpha, h_{1}\right] \times[-\beta, b]$. We denote by $u_{1}^{*}$ the restriction of this unique solution on $\left[0, h_{1}\right] \times[0, b]$.

Next we consider the area $\left[h_{1}, h_{2}\right] \times[0, b]$. We define a closed subset $C_{h_{2}} \subset C\left(\left[-\alpha, h_{2}\right] \times[-\beta, b] ; \mathbb{R}^{n}\right)$ which contains those functions $u$ that $u(x, y)=\phi(x, y)$ for $(x, y) \in \widetilde{J}$, $u(x, y)=u_{1}^{*}(x, y)$ for $(x, y) \in\left[0, h_{1}\right] \times[0, b]$ and the restriction of $u$ on $\left[h_{1}, h_{2}\right] \times[0, b]$ is continuous. Let us define an operator, also denoted by $N$, from $C_{h_{2}}$ into itself as

$$
(N u)(x, y)= \begin{cases}\phi(x, y), & (x, y) \in \tilde{J}, \\ u_{1}^{*}(x, y), & (x, y) \in\left[0, h_{1}\right] \times[0, b], \\ g(x, y)+\frac{1}{\Gamma\left(r_{1}\right) \Gamma\left(r_{2}\right)} \int_{h_{1}}^{x} \int_{0}^{y} f\left(s, t, u_{(s, t)}\right)(x-s)^{r_{1}-1}(y-t)^{r_{2}-1} d t d s, & (x, y) \in\left[h_{1}, h_{2}\right] \times[0, b],\end{cases}
$$

where

$$
\begin{aligned}
& g(x, y)=\mu(x, y)+\frac{1}{\Gamma\left(r_{1}\right) \Gamma\left(r_{2}\right)} \\
& \quad \cdot \int_{0}^{h_{1}} \int_{0}^{y}(x-s)^{r_{1}-1}(y-t)^{r_{2}-1} f\left(s, t, u_{(s, t)}\right) d t d s .
\end{aligned}
$$

Since $u(x, y)=u_{1}^{*}(x, y)$ for $(x, y) \in\left[0, h_{1}\right] \times[0, b]$ is known, $g$ is a known function. Using the same arguments as above we obtain that there is a unique fixed point of $N$ on $C_{h_{2}}$. We denote by $u_{2}^{*}$ the restriction of this unique function on $\left[h_{1}, h_{2}\right] \times[0, b]$.

Take the next area $\left[h_{2}, h_{3}\right] \times[0, b]$ and, repeating this process, we obtain $u_{1}^{*}, u_{2}^{*}, \ldots, u_{N}^{*}$ defined on $\left[0, h_{1}\right] \times$ $[0, b],\left[h_{1}, h_{2}\right] \times[0, b], \ldots,\left[h_{N-1}, a\right] \times[0, b]$, respectively. Define a function $u:[-\alpha, a] \times[-\beta, b] \rightarrow \mathbb{R}^{n}$ as

$$
u(x, y)= \begin{cases}\phi(x, y), & (x, y) \in \widetilde{J} \\ u_{1}^{*}(x, y), & (x, y) \in\left[0, h_{1}\right] \times[0, b], \\ u_{2}^{*}(x, y), & (x, y) \in\left[h_{1}, h_{2}\right] \times[0, b], \\ \vdots & \\ u_{N}^{*}(x, y), & (x, y) \in\left[h_{N-1}, a\right] \times[0, b] .\end{cases}
$$

It is easy to check that $u$ is the unique solution of (1)-(3) on $[-\alpha, a] \times[-\beta, b]$, and this completes the proof.
Remark 11. In Theorem 10 there is no restriction to the Lipschitz constant, from which it seems that the assumption $k a^{r_{1}} b^{r_{2}} / \Gamma\left(r_{1}+1\right) \Gamma\left(r_{2}+1\right)<1$ in [1, Theorem 3.2] may not be necessary.

Using the same argument as in Theorem 10, we can prove an existence result for the following initial value problem without delay:

$$
\begin{aligned}
&\left({ }^{c} D_{0}^{r} u\right)(x, y)=g(x, y, u(x, y)), \\
& \quad(x, y) \in J:=[0, a] \times[0, b], \\
& u(x, 0)=\varphi(x), \quad x \in[0, a], \\
& u(0, y)=\psi(y), \quad y \in[0, b],
\end{aligned}
$$

where $g: J \times \mathbb{R}^{n} \rightarrow \mathbb{R}^{n}$ is a given function and $\varphi:[0, a] \rightarrow$ $\mathbb{R}^{n}, \psi:[0, b] \rightarrow \mathbb{R}^{n}$ are given absolutely continuous functions with $\varphi(0)=\psi(0)$.

Theorem 12. Assume that $g: J \times \mathbb{R}^{n} \rightarrow \mathbb{R}^{n}$ is continuous and is Lipschitz continuous with respect to the last variable; that is, there is $k>0$ such that

$$
\|g(x, y, u)-g(x, y, v)\| \leq k\|u-v\|
$$

for any $u, v \in \mathbb{R}^{n}$ and $(x, y) \in J$. Then there exists a unique solution to $(34)$ on $[0, a] \times[0, b]$. 
Next we prove a continuous dependent result with respect to the order of the fractional derivative. For simplicity, we consider the case that $\widetilde{r}=\left(\widetilde{r}_{1}, \widetilde{r}_{2}\right)$ tends to $r=\left(r_{1}, r_{2}\right) \in$ $(0,1] \times(0,1]$ monotonically, that is, $\widetilde{r}_{1} \uparrow r_{1}$ and $\widetilde{r}_{2} \uparrow r_{1}$ simultaneously. We denote this case by $\widetilde{r} \uparrow r$.

Theorem 13. Let $u$ be the solution of problem (1)-(3) and $v$ the solution of the problem

$$
\begin{aligned}
\left({ }^{c} D_{0}^{\tilde{r}} v\right)(x, y) & =f(x, y, v(x, y)), \\
(x, y) \in J:=[0, a] \times[0, b], & \\
v(x, y) & =\phi(x, y), \\
(x, y) & \in \widetilde{J}:=[-\alpha, a] \times[-\beta, b] \backslash(0, a] \times(0, b], \\
v(x, 0) & =\varphi(x), \quad x \in[0, a], \\
v(0, y) & =\psi(y), \quad y \in[0, b] .
\end{aligned}
$$

$$
\begin{aligned}
& \|u(x, y)-v(x, y)\| \leq\left(\frac{1}{\Gamma\left(r_{1}\right) \Gamma\left(r_{2}\right)}-\frac{1}{\Gamma\left(\widetilde{r}_{1}\right) \Gamma\left(\widetilde{r}_{2}\right)}\right) \int_{0}^{x} \int_{0}^{y}(x-s)^{r_{1}-1}(y-t)^{r_{2}-1}\left\|f\left(s, t, u_{(s, t)}\right)\right\| d t d s \\
& +\frac{1}{\Gamma\left(\widetilde{r}_{1}\right) \Gamma\left(\widetilde{r}_{2}\right)}\left[\int_{0}^{x} \int_{0}^{y}\left[(x-s)^{r_{1}-1}(y-t)^{r_{2}-1}-(x-s)^{\tilde{r}_{1}-1}(y-t)^{\tilde{r}_{2}-1}\right]\left\|f\left(s, t, u_{(s, t)}\right)\right\| d t d s\right. \\
& \left.\quad+\int_{0}^{x} \int_{0}^{y}(x-s)^{\widetilde{r}_{1}-1}(y-t)^{\tilde{r}_{2}-1}\left\|f\left(s, t, u_{(s, t)}\right)-f\left(s, t, v_{(s, t)}\right)\right\| d t d s\right] \leq\left(\frac{1}{\Gamma\left(r_{1}\right) \Gamma\left(r_{2}\right)}-\frac{1}{\Gamma\left(\widetilde{r}_{1}\right) \Gamma\left(\widetilde{r}_{2}\right)}\right) \frac{M a^{r_{1}} b^{r_{2}}}{r_{1} r_{2}} \\
& \quad+\frac{M}{\Gamma\left(\widetilde{r}_{1}\right) \Gamma\left(\widetilde{r}_{2}\right)}\left[\frac{x^{r_{1}} y^{r_{2}}}{r_{1} r_{2}}-\frac{x^{\tilde{r}_{1}} y^{\tilde{r}_{2}}}{\widetilde{r}_{1} \widetilde{r}_{2}}\right]+\frac{k}{\Gamma\left(\widetilde{r}_{1}\right) \Gamma\left(\widetilde{r}_{2}\right)} \int_{0}^{x} \int_{0}^{y}(x-s)^{\widetilde{r}_{1}-1}(y-t)^{\widetilde{r}_{2}-1}\left\|u_{(s, t)}-v_{(s, t)}\right\|_{C} d t d s .
\end{aligned}
$$

Noting that $u(x, y)=v(x, y)=\phi(x, y)$ for $(x, y) \in \widetilde{J}$, it follows that

$$
\begin{aligned}
& \|u(s, t)-v(s, t)\|_{C} \\
& =\sup _{(\sigma, \tau) \in[-\alpha, 0] \times[-\beta, 0]}\|u(s+\sigma, t+\tau)-v(s+\sigma, t+\tau)\| \\
& =\sup _{(\sigma, \tau) \in[0, s] \times[0, t]}\|u(\sigma, \tau)-v(\sigma, \tau)\| .
\end{aligned}
$$

Since $r$ is fixed and $\max \left\{r_{1}-\widetilde{r}_{1}, r_{2}-\widetilde{r}_{2}\right\}<\varepsilon$ is sufficiently small, we can find $\delta>0$ such that $r_{1}, r_{2}, \widetilde{r}_{1}, \widetilde{r}_{2}>\delta$. Based on the fact that gamma function $\Gamma(\cdot)$ is continuously differentiable on $[\delta, 1]$ and $\Gamma^{\prime}(\cdot)$ is bounded on $[\delta, 1]$, we can find a constant $K_{1}>0$ such that

$$
\left|\frac{1}{\Gamma\left(r_{1}\right) \Gamma\left(r_{2}\right)}-\frac{1}{\Gamma\left(\widetilde{r}_{1}\right) \Gamma\left(\widetilde{r}_{2}\right)}\right| \leq K_{1} \varepsilon .
$$

Further, we can find constant $K_{2}>0$ such that

$$
\left|\frac{x^{r_{1}} y^{r_{2}}}{r_{1} r_{2}}-\frac{x^{\tilde{r}_{1}} y^{\widetilde{r}_{2}}}{\widetilde{r}_{1} \widetilde{r}_{2}}\right| \leq K_{2} \varepsilon
$$

Suppose that the hypotheses of Theorem 10 hold. Then

$$
\lim _{\widetilde{r} \uparrow r(x, y) \in J} \max _{\|u(x, y)-v(x, y)\|=0}
$$

Proof. Existence and uniqueness of the solutions can be deduced by Theorem 10. Let

$$
M=\sup _{(x, y) \in J}\left\{\left\|f\left(x, y, u_{(x, y)}\right)\right\|,\left\|f\left(x, y, v_{(x, y)}\right)\right\|\right\} .
$$

It follows from the continuity of $u, v$, and $f$ on closed bounded domains that $M<+\infty$. By Definition 9, $u$ and $v$ can be expressed as the form of (23). Let $\varepsilon>0$ be given and $\max \left\{r_{1}-\widetilde{r}_{1}, r_{2}-\widetilde{r}_{2}\right\}<\varepsilon$. Then, for $(x, y) \in J$, we have
It then follows from (39)-(42) that

$$
\begin{aligned}
& \|u(x, y)-v(x, y)\| \leq K \varepsilon+\frac{k}{\Gamma\left(\widetilde{r}_{1}\right) \Gamma\left(\widetilde{r}_{2}\right)} \\
& \cdot \int_{0}^{x} \int_{0}^{y}(x-s)^{\widetilde{r}_{1}-1}(y-t)^{\tilde{r}_{2}-1} \\
& \cdot \sup _{(\sigma, \tau) \in[0, s] \times[0, t]}\|u(\sigma, \tau)-v(\sigma, \tau)\| d t d s \leq K \varepsilon \\
& +\frac{k}{\Gamma\left(r_{1}\right) \Gamma\left(r_{2}\right)} \int_{0}^{x} \int_{0}^{y}(x-s)^{r_{1}-1}(y-t)^{r_{2}-1} \\
& \cdot \sup _{(\sigma, \tau) \in[0, s] \times[0, t]}\|u(\sigma, \tau)-v(\sigma, \tau)\| d t d s,
\end{aligned}
$$

where

$$
K=\frac{K_{1} M a^{r_{1}} b^{r_{2}}}{r_{1} r_{2}}+\frac{K_{2} M}{\Gamma\left(r_{1}\right) \Gamma\left(r_{2}\right)} .
$$

Define a function $\omega(\cdot, \cdot):[0, a] \times[0, b] \rightarrow \mathbb{R}$ as

$$
\omega(x, y)=\sup _{(\sigma, \tau) \in[0, x] \times[0, y]}\|u(\sigma, \tau)-v(\sigma, \tau)\| .
$$


Take supremun on both sides of (43); we get that

$$
\begin{aligned}
& \omega(x, y) \leq K \varepsilon+\frac{k}{\Gamma\left(r_{1}\right) \Gamma\left(r_{2}\right)} \\
& \quad \cdot \int_{0}^{x} \int_{0}^{y}(x-s)^{r_{1}-1}(y-t)^{r_{2}-1} \omega(s, t) d t d s .
\end{aligned}
$$

An application of Lemma 7 yields that

$$
\begin{aligned}
& \omega(x, y) \\
& \leq K \varepsilon \\
& \quad+\frac{k K \delta^{\prime} \varepsilon}{\Gamma\left(r_{1}\right) \Gamma\left(r_{2}\right)} \int_{0}^{x} \int_{0}^{y}(x-s)^{r_{1}-1}(y-t)^{r_{2}-1} d t d s \\
& \leq\left(K+\frac{k K \delta^{\prime} a^{r_{1}} b^{r_{2}}}{\Gamma\left(r_{1}+1\right) \Gamma\left(r_{2}+1\right)}\right) \varepsilon,
\end{aligned}
$$

where $\delta^{\prime}$ is a constant dependent on $r_{1}$ and $r_{2}$. This concludes the proof.

\section{Examples}

In this section we discuss some examples as applications of our results. We first consider the partial hyperbolic functional differential equations of the form

$$
\begin{aligned}
\left({ }^{c} D_{0}^{r} u\right)(x, y) & =\frac{100}{e^{x+y+2}(1+|u(x-1, y-2)|)}, \\
& (x, y) \in[0,1] \times[0,1], \\
u(x, y) & =x+y^{2}, \quad x \in[0,1], \\
(x, y) \in[-1,1] \times[-2,1] \backslash(0,1] \times(0,1], & \\
u(x, 0) & =x, \quad y \in[0, b],
\end{aligned}
$$

and we set

$$
\begin{array}{r}
f\left(x, y, u_{(x, y)}\right)=\frac{100}{e^{x+y+2}(1+|u(x-1, y-2)|)}, \\
(x, y) \in[0,1] \times[0,1] .
\end{array}
$$

Then for each $u, \bar{u} \in C$ and $(x, y) \in[0,1] \times[0,1]$, we have

$$
\left|f\left(x, y, u_{(x, y)}\right)-f\left(x, y, \bar{u}_{(x, y)}\right)\right| \leq \frac{100}{e^{2}}\|u-\bar{u}\|_{C}
$$

that is, $f$ is Lipschitz continuous with respect to the last variable with Lipschitz constant $k=100 / e^{2}$. We derive from Theorem 10 that problem (48) has a unique solution defined on $[-1,1] \times[-2,1]$. A similar example is discussed on page 32 in [1]. We indicate that the restriction on the Lipschitz constant there is not necessary.
We now consider the boundary value problem

$$
\begin{aligned}
\left({ }^{c} D_{0}^{r} u\right)(x, y) & =f(x, y) u^{2}(x, y), \\
\qquad(x, y) \in[0, a] \times[0, b], & \\
u(x, 0) & =x, \quad x \in[0, a], \\
u(0, y) & =y^{2}, \quad y \in[0, b],
\end{aligned}
$$

where $f$ can be any given continuous function defined on $[0, a] \times[0, b]$. The solution to $(51)$ has the form

$$
\begin{aligned}
& u(x, y)=x+y^{2}+\frac{1}{\Gamma\left(r_{1}\right) \Gamma\left(r_{2}\right)} \\
& \quad \cdot \int_{0}^{x} \int_{0}^{y}(x-s)^{r_{1}-1}(y-t)^{r_{2}-1} f(s, t) u^{2}(s, t) d t d s .
\end{aligned}
$$

Since a continuous function is bounded on a closed bounded region, it is easy to see that the assumption of Theorem 12 holds. Consequently, Theorem 12 implies that problem (51) has a unique solution on $[0, a] \times[0, b]$.

\section{Conflict of Interests}

The authors declare that they have no competing interests.

\section{Acknowledgments}

The authors are grateful to the reviewers for a painstaking reading of this paper and for their valuable suggestions on improving this paper. This work was supported by the National Natural Science Foundation of China (11271316, 11571300, and 11201410) and the Natural Science Foundation of Jiangsu Province (BK2012260).

\section{References}

[1] S. Abbas, M. Benchohra, and G. M. N’Guérékata, Topics in Fractional Differential Equations, vol. 27 of Developments in Mathematics, Springer, New York, NY, USA, 2012.

[2] D. Delbosco and L. Rodino, "Existence and uniqueness for a nonlinear fractional differential equation," Journal of Mathematical Analysis and Applications, vol. 204, no. 2, pp. 609-625, 1996.

[3] K. Diethelm, The Analysis of Fractional Differential Equations, vol. 2004 of Lecture Notes in Mathematics, Springer, Berlin, Germany, 2010.

[4] R. Hilfer, "Experimental evidence for fractional time evolution in glass forming materials," Chemical Physics, vol. 284, no. 1-2, pp. 399-408, 2002.

[5] R. Hilfer, "On fractional relaxation," Fractals: Complex Geometry, Patterns, and Scaling in Nature and Society, vol. 11, supplement 1, pp. 251-257, 2003.

[6] A. A. Kilbas, H. M. Srivastava, and J. J. Trujillo, Theory and Applications of Fractional Differential Equations, vol. 204 of North-Holland Mathematics Studies, Elsevier Science, Amsterdam, The Netherlands, 2006.

[7] V. V. Uchaikin, Fractional Derivatives for Physicists and Engineers: Volume I Background and Theory Volume II Applications, Springer, Berlin, Germany, 2013. 
[8] A. Alsaedi, S. K. Ntouyas, R. P. Agarwal, and B. Ahmad, "On Caputo type sequential fractional differential equations with nonlocal integral boundary conditions," Advances in Difference Equations, vol. 2015, article 33, 12 pages, 2015.

[9] L. Chen, Z. Fan, and G. Li, "On a nonlocal problem for fractional differential equations via resolvent operators," Advances in Difference Equations, vol. 2014, article 251, pp. 1-12, 2014.

[10] M. A. Darwish and S. K. Ntouyas, "On initial and boundary value problems for fractional order mixed type functional differential inclusions," Computers \& Mathematics with Applications, vol. 59, no. 3, pp. 1253-1265, 2010.

[11] Q. Dong, "Existence and continuous dependence for weighted fractional differential equations with infinite delay," Advances in Difference Equations, vol. 2014, article 190, pp. 1-13, 2014.

[12] X. Wang, L. Wang, and Q. Zeng, "Fractional differential equations with integral boundary conditions," Journal of Nonlinear Science and Its Applications, vol. 8, no. 4, pp. 309-314, 2015.

[13] Y. Zhou, "Recent advances in fractional differential equations," Applied Mathematics and Computation, vol. 257, p. 1, 2015.

[14] S. Abbas, M. Benchohra, and J. J. Nieto, "Global uniqueness results for fractional order partial hyperbolic functional differential equations," Advances in Difference Equations, vol. 2011, Article ID 379876, 25 pages, 2011.

[15] D. Henry, Geometric Theory of Semilinear Parabolic Partial Differential Equations, Springer, Berlin, Germany, 1989. 


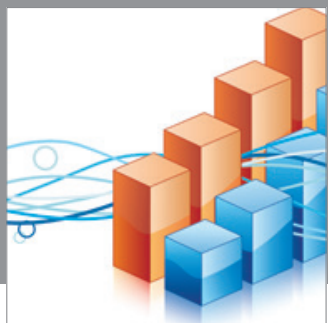

Advances in

Operations Research

mansans

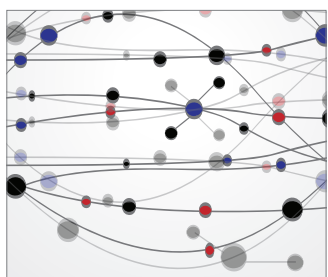

The Scientific World Journal
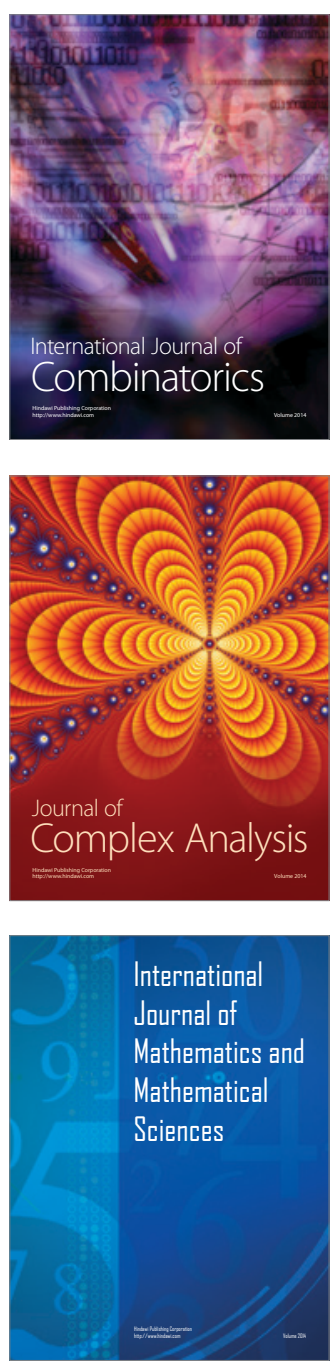
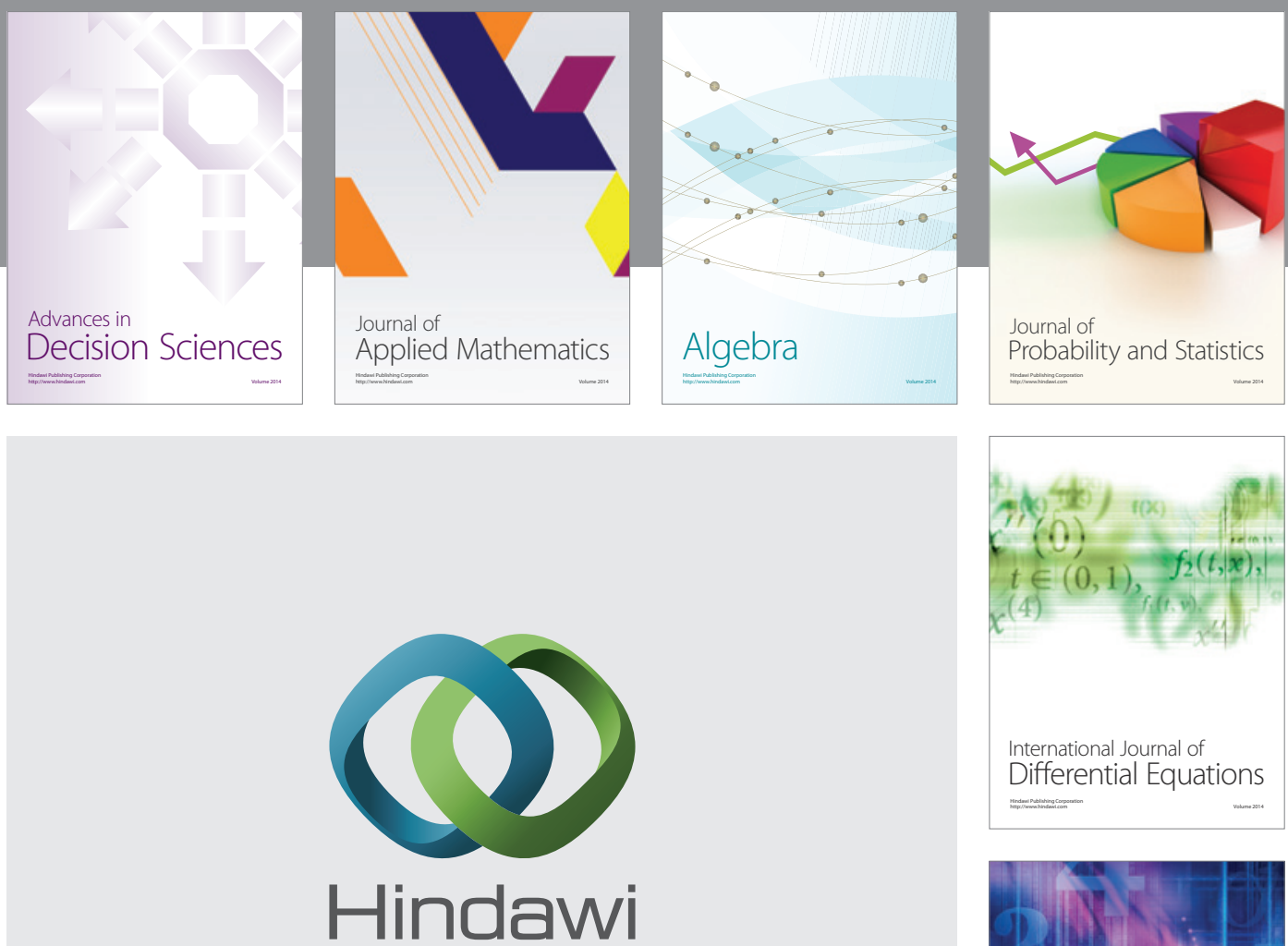

Submit your manuscripts at http://www.hindawi.com
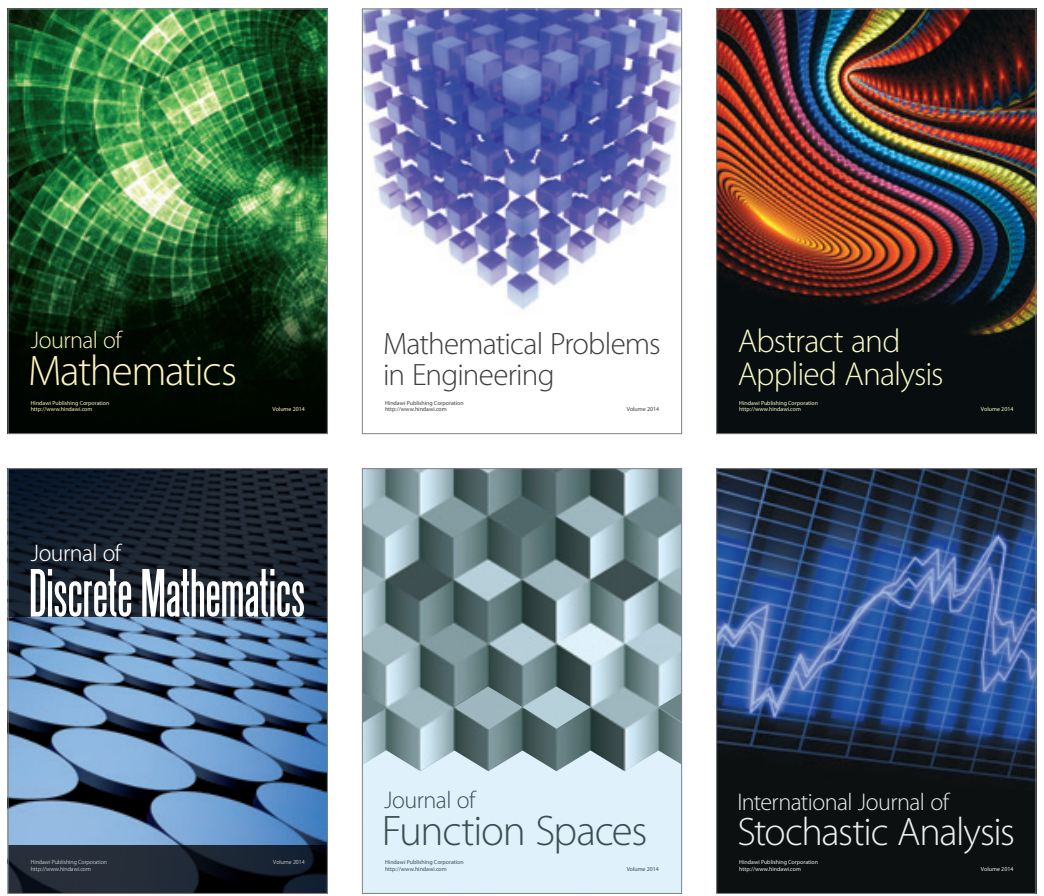

Journal of

Function Spaces

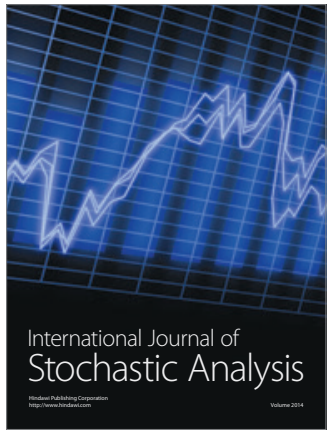

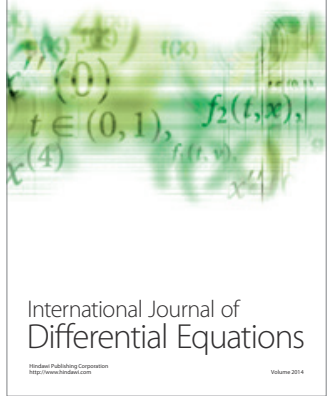
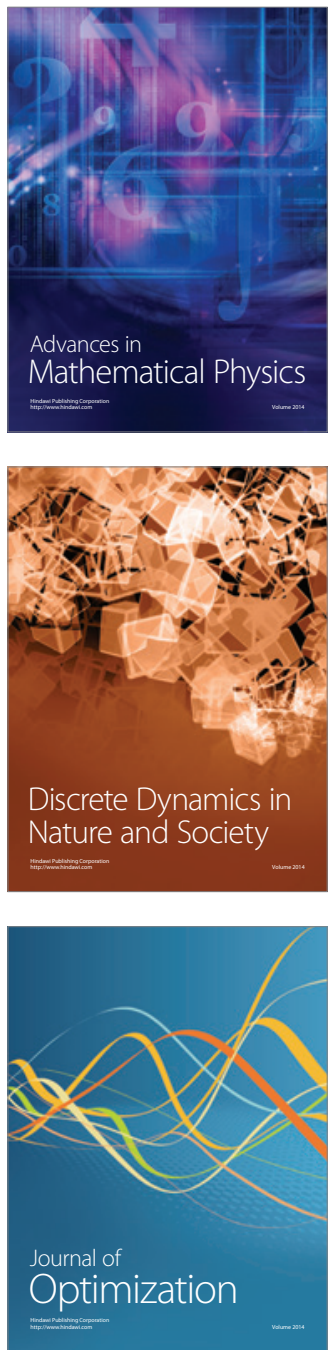